\title{
Time-Frequency Analysis of Nonstationary Optical Signals Using Husimi Type Function
}

\author{
Miloš D. Davidovićc ${ }^{a, *}$ Milena D. Davidović ${ }^{b}$ And Vuk Vojisavluevic ${ }^{c}$ \\ ${ }^{a}$ School of Electrical Engineering, University of Belgrade, Serbia \\ ${ }^{b}$ Faculty of Civil Engineering, University of Belgrade, Serbia \\ ${ }^{c}$ School of Electrical and Computer Engineering, RMIT University, Melbourne, Australia
}

\begin{abstract}
Time-frequency analysis is important to identify the localized information of a non-stationary signal in the time and frequency domains simultaneously. There are few different time-frequency analysis methods available with their own specialty and suitability. In quantum mechanics Husimi function of any quantum mechanical state arises naturally whenever the simultaneous measurement of both coordinate and momentum is performed on this state with maximal accuracy allowed by the Heisenberg uncertainty relations. The Husimi function is a probability distribution for the statistics of simultaneous unsharp measurement of both coordinate and momentum. In general, like the Wigner function, the Husimi function can be defined in the space of any pair of conjugate variables. In particular, in the studies of signal processing, this space is the time-frequency space. In the present work we consider the Husimi function in this space, and apply it to analyse the multicomponent signals. The time-frequency representations of the simulated signals by using the Husimi distribution clearly show the frequency features along the time axis. The results are encouraging and indicate that, like in corresponding analogous problems in quantum mechanics, the Husimi distribution approach in the time-frequency analysis for non-stationary optical signals may provide some insights which are not so easily obtained in other, more spread approaches.
\end{abstract}

PACS numbers: 43.60.Hj

\section{Introduction}

Many natural signals are non-stationary, i.e., their frequency components evolve over time. For those signals, the popularly used Fourier transform is not adequate to reveal the frequency content which is localized in time, e.g., the high-frequency bursts. The timefrequency analysis (TFA) decomposes a time-domain signal into a time and frequency two-dimensional space in which the localized information can be presented. The spectrogram obtained by the time-frequency analysis is generally more desirable than the spectrum obtained by the Fourier transform.

A few time-frequency analysis approaches have been developed, namely the short time Fourier transform (STFT), the Wigner-Ville distribution (WVD), the wavelet transform, etc. In the STFT, the signal is isolated in a "sliding window", then the fast Fourier transform (FFT) analysis is performed to estimate the "local" frequency content. The Wigner-Ville distribution was first defined by Wigner in the context of quantum mechanics [1], and later independently introduced by Ville for signal processing and spectral analysis [2]. Though the Wigner-Ville distribution gives the high resolution in the time-frequency domain, it is not used widely for

* corresponding author; e-mail: milos.d.davidovic@gmail.com practical applications due to the interaction between different signal components, the so-called "cross term" [3]. The wavelet transforms, unlike the Fourier transforms which use sines and cosines, use the basis functions of limited duration to decompose a signal [4]. Wavelets are well-suited for approximating data with sharp discontinuities. However, the wavelet transform also suffers a few drawbacks, e.g., it does not provide the shift-invariant property, and sometimes to choose appropriate wavelet could be problematic while processing practical signals and custom designed wavelets are required [5].

In an analogous way as the Wigner function, the Husimi function also can be defined for other pairs of conjugate variables, such as time and frequency. There are important elements of formal mathematical correspondence between quantum mechanics and signal analysis. Historically, research work on joint time-frequency distributions has often been inspired and guided by corresponding quantum mechanical approaches, in spite of essential physical differences of both fields. By this historical analogy, it is worth to investigate to which extent the Husimi function in this new signal analysis context, and new time frequency space, inherits its most essential feature from quantum mechanics and ordinary $(q, p)$ phase space. It is well known that the knowledge of the exact (sharp) quantum mechanical probability distributions both for coordinate and momentum does not supply enough information to determine, in a unique way, a 
quantum state which has such probability distributions of coordinate and momentum. On the other hand, the essential feature of the Husimi function, which can be interpreted as the probability distribution obtained from simultaneous optimal unsharp measurement of coordinate and momentum, which are canonically conjugated variables, is that it contains the complete information about the corresponding quantum state. Namely, by inverting the Husimi function, this quantum mechanical state can be completely determined in the unique way. In what follows, we investigate the properties of the Husimi function on several concrete characteristic examples.

\section{Time-frequency Husimi distribution function Wigner and Husimi functions in signal processing}

Both the Wigner and Husimi functions are the phase space quasidistributions in quantum mechanics. These functions provide a two-dimensional picture of a one-dimensional wave function, and can be compared directly with classical phase space distributions [6]. Recently, they have been used in the study of quantum information, a new research field in which quantum mechanics is used to process and manipulate information [7-9].

In quantum mechanics, coordinate and momentum are conjugate variables. In signal analysis, time and frequency are corresponding conjugate variables. Apart from the absence of quantum mechanical Planck constant, definitions and relations of Wigner and Husimi functions follow the same way as in quantum mechanical case. So the Wigner function of a function $f$, say, is

$$
W(t, \omega)=\frac{1}{\pi} \int_{-\infty}^{\infty} \mathrm{d} \tau \mathrm{e}^{-2 \mathrm{i} \varpi \tau} f^{*}(t-\tau) f(t+\tau) .
$$

In time-frequency space its Gaussian smoothing is given by

$$
\begin{aligned}
& G(t, \varpi)=\frac{1}{2 \pi \sigma_{t} \sigma_{\varpi}} \int \mathrm{d} t^{\prime} \int \mathrm{d} \varpi^{\prime} \exp \left(-\frac{\left(t^{\prime}-t\right)^{2}}{2 \sigma_{t}^{2}}\right) \\
& \quad \times \exp \left(-\frac{\left(\varpi^{\prime}-\varpi\right)^{2}}{2 \sigma_{\varpi}^{2}}\right) W\left(t^{\prime}, \varpi^{\prime}\right) .
\end{aligned}
$$

When the widths $\sigma_{t}$ and $\sigma_{\varpi}$ are such that $\sigma_{t} \sigma_{\varpi}=\frac{1}{2}$, the above Gaussian becomes the time-frequency Husimi distribution (HD) function

$$
H(t, \varpi)=\frac{1}{2 \pi} \sqrt{\frac{\kappa}{\pi}}\left|\int \mathrm{e}^{-\kappa(\tau-t)^{2} / 2} \mathrm{e}^{-\mathrm{i} \varpi t} f(\tau) \mathrm{d} \tau\right|^{2}
$$

in full analogy with quantum mechanical case, and where now $\kappa=\frac{1}{2 \sigma_{t}^{2}}=2 \sigma_{\varpi}^{2}$. The values of $\sigma_{\varpi}$ and $\sigma_{t}$ are the applications dependent on the HD where they balance between the resolutions in frequency and time domains.

\section{Results and discussion}

In this section, we investigate the performance of the $\mathrm{HD}$ in the analysis of multicomponent non-stationary signals.

\subsection{Analysis of a Gaussian pulse}

We analyse the HD of a Gaussian pulse signal shifted in frequency spectrum by $f_{0}=5 \times 10^{14} \mathrm{~Hz}$

$$
x(t)=A \exp \left(-\frac{\left(t-t_{0}\right)^{2}}{2 \sigma^{2}}\right) \sin \left(\omega_{0} t\right),
$$

where $\omega_{0}=2 \pi f_{0}$ is the angular frequency.
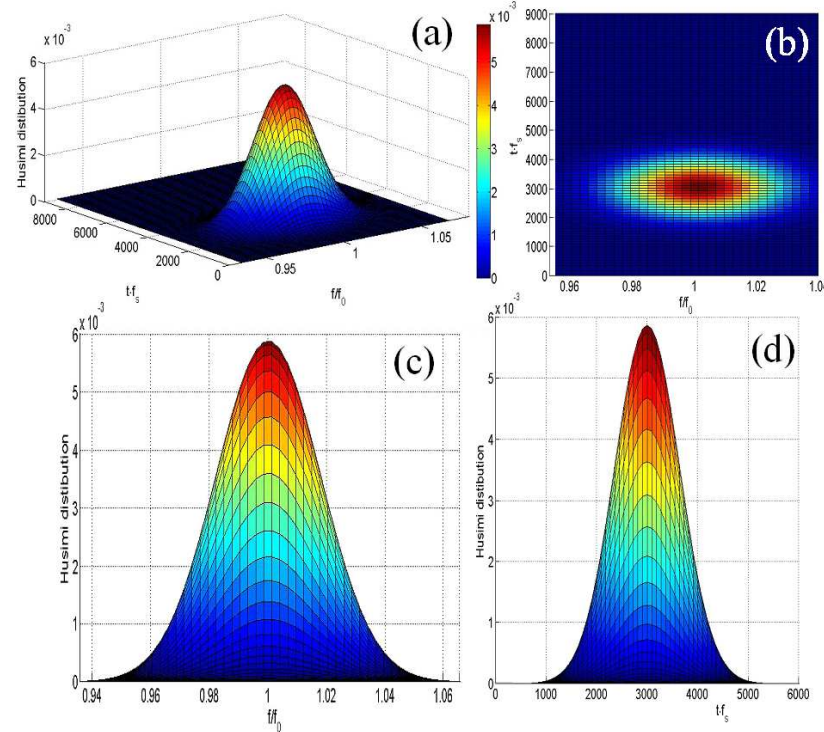

Fig. 1. Husimi distribution of shifted Gaussian pulse (2) with $A=1, t_{0}=1 \mathrm{~s}, \sigma=0.3 \mathrm{~s}$ : (a) $3 \mathrm{D}$ view, (b) $x y$ view, (c) $x z$ view, (d) $y z$ view.

From Fig. 1 we can see that the HD of the Gausian pulse in the time domain given by (2) becomes the Gaussian pulse in the frequency domain centered about $f_{0}$ as expected (Fig. 1c).

\subsection{Analysis of sinusoidal pulses shifted in time}

We analyse the HD of a multicomponent signal given by

$$
\begin{aligned}
& x(t)=p\left(\frac{t}{t_{p 1}}\right) \sin \left(\omega_{1} t\right)+p\left(\frac{t-t_{\text {delay }}}{t_{p 2}}\right) \sin \left(\omega_{2} t\right) \\
& +1.2 p\left(\frac{t}{t_{p 3}}\right) \sin \left(\omega_{3} t\right)+1.2 p\left(\frac{t-t_{\text {delay }}}{t_{p 4}}\right) \sin \left(\omega_{4} t\right),
\end{aligned}
$$

where $p$ denotes the pulse function given by

$$
p(x)= \begin{cases}1, & 0<x<1 \\ 0 & \text { otherwise }\end{cases}
$$

the values of used time constants are $t_{p 1}=t_{p 3}=2 \mathrm{~s}$, $t_{p 2}=t_{p 4}=1 \mathrm{~s}, t_{\text {delay }}=1 \mathrm{~s}$ and $\omega_{i}=2 \pi f_{i}$ are angular frequencies.

We can see from Fig. 2 that the components of higher amplitude in the time domain correspond to higher "hills" in the HD. It is also noticeable that components that begin later in time are translated along the time axis in the HD plot. The peaks along the frequency axis are centred around the frequencies of the signal, but they 
have certain width that can be adjusted using different values of $\sigma_{t}$. By increasing $\sigma_{t}$ we get less precise HD representation of the signal along the time axis but we get sharper spikes along the frequency axis and vice versa. Particular choice of $\sigma_{t}$ depends on application.

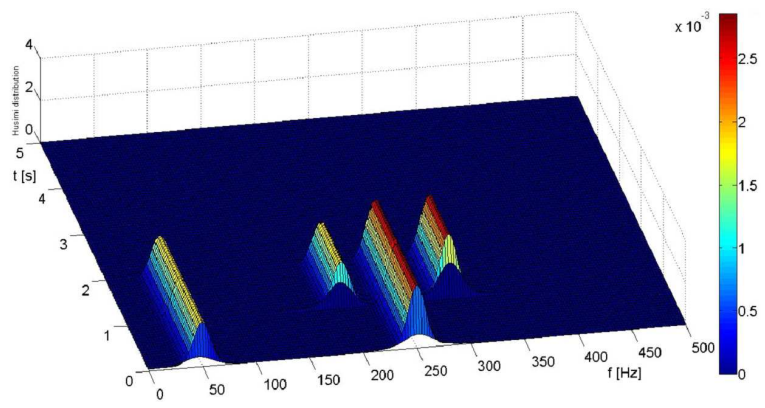

Fig. 2. The HD of a multicomponent signal given by (4) with $f_{1}=50 \mathrm{~Hz}, f_{2}=200 \mathrm{~Hz}, f_{3}=250 \mathrm{~Hz}$, $f_{4}=300 \mathrm{~Hz}$

\section{Conclusions}

In this paper a time-frequency analysis was performed, which was based on a function, Husimi distribution, popularly used in quantum physics. HD provides a good time-frequency representation by adjusting the shape of its kernel, through the controlling parameter $\sigma_{t}$, to optimally pass auto-components and move cross-components, regardless of their location and orientation in the timefrequency plane. It is shown that the HD convenes most of the desirable properties with high time frequency resolution.

Simulation results presented in this paper indicate that the used distribution, HD, is a promising approach in the time-frequency analysis. Also, this study, in a way, reveals the potentials of the HD distribution in multicomponent signal analysis.

\section{Acknowledgments}

Miloš D. Davidović and Milena Davidović acknowledge the support from the Ministry of Science of Serbia, under the contracts $141041 \mathrm{G}$ and 141003 , respectively.

\section{References}

[1] E.P. Wigner, Phys. Rev. 40, 749 (1932).

[2] J. Ville, Cables et Transmission 2A, 61 (1948).

[3] L. Cohen, Time-Frequency Analysis, Prentice Hall PTR, Englewood Cliffs, New Jersey 1995.

[4] I. Daubechies, IEEE Trans. Info Theory 36, 961 (1990).

[5] W. Sweldens, Appl. Comput. Harmon. Anal. 3, 186 (1996).

[6] D.M. Davidović, D. Lalović, Physica A 182, 643 (1992).

[7] P. Leboeuf, A. Voros, J. Phys. A, Math. Gen. 23 1765 (1990).

[8] S.L. Braunstein, V. Budek, M. Hillery, quant-ph/ 0009076 v1, September 2000.

[9] D.M. Appleby, J. Mod. Opt. 46, 825 (1999). 